\title{
Optimization of electroacoustic absorbers by means of designed experiments
}

\author{
R. Boulandet* , H. Lissek \\ Laboratory of Electromagnetics and Acoustics (LEMA), École Polytechnique Fédérale de Lausanne (EPFL), Station 11, CH-1015 Lausanne, Switzerland
}

\section{A R T I C L E I N F O}

\section{Article history:}

Received 10 February 2010

Received in revised form 26 April 2010

Accepted 27 April 2010

Available online 9 June 2010

\section{Keywords:}

Electroacoustic absorber

Acoustic impedance matching

Optimization design

Response surface methodology

Design of experiments

\begin{abstract}
A B S T R A C T
In multivariate systems, when it comes to identifying actual operating conditions ranges, or optimal settings, the use of constrained optimization is often required. Among the different tools for the engineer to perform such optimization, designed experiments offer accurate performances. In this paper, the optimization process of "electroacoustic absorbers" is investigated by means of response surface methodology. A multivariate linear model is established by a series of designed experiments in order to analyze the modification of electroacoustic absorber performances due to the variation of several constitutive parameters (such as the moving mass of the loudspeaker, the enclosure volume, the filling density of mineral fiber within the enclosure, and the electrical load value to which the loudspeaker is connected), that influence their whole absorbing mechanisms. A simple case study is then provided to illustrate the capabilities of the developed optimization procedure, from which general conclusions on such design methodology, as well as on electroacoustic absorbers sensitivity, are drawn.
\end{abstract}

(c) 2010 Elsevier Ltd. All rights reserved.

\section{Introduction}

Electroacoustic absorbers are semi-active, or eventually active, devices dedicated to noise reduction in the low-frequency range. Basically, such devices consist of a loudspeaker system, including acoustic circuits and enclosures (totally or partially filled with porous material), the whole constituting a resonant system capable of absorbing sound energy within a frequency range in which conventional passive materials are not effective and/or cumbersome. When connecting a resistive load of positive value to the electrical terminals, one can significantly modify the value of the acoustic impedance that the transducer presents to the external environment, in a semi-active manner [2,3]. Thus, by selecting a suitable resistive load the acoustic impedance of the electroacoustic absorber can be tuned so as to match the characteristic impedance of air, and hence the transducer becomes then even more absorbent around its resonance frequency. When it comes to integration however, some design parameters, such as enclosure volume if overall dimensions are limited, are generally specified, which corresponds to a constraint in view of finding out the best compromise in terms of absorption capabilities versus physical embodiment of the device. For instance, in the frame of lowfrequency noise control in rooms, the damping of first modal frequencies requires very bulky devices (such as bass-traps or panel absorbers), to which such optimized electroacoustic absorbers with quite low overall dimensions could represent an interesting alternative [4]. Consequently, the overall mechanisms exhibited

\footnotetext{
* Corresponding author.

E-mail address: romain.boulandet@epfl.ch (R. Boulandet).
}

by an electroacoustic absorber are a combination of dissipative effects due to the mechanical losses in the loudspeaker's moving body, viscous dissipation of air induced by the penetration of sound waves in a porous medium filling the enclosure, and the dissipation in form of thermal energy induced by the circulation of electrical current within the resistive load connected to the terminals of the loudspeaker.

Although many authors have conducted research on the design of electroacoustic absorbers based on shunt electrodynamic loudspeakers [2] or electromechanical Helmholtz resonators [3], only few of them have reported the optimization study of absorbing/ reflecting performances. Regarding the design of acoustic absorbers, some references addressing optimization processes may be found in the literature. In their work, Yu et al. describe an analytical solution of a resonator-enclosure interaction model to optimize the resonator resistance [5]. It is shown that the proposed model serves as an efficient design tool to determine the internal resistance of the Helmholtz resonator in order to achieve optimal sound reduction in the frequency band comprising acoustic resonances. More recently, Ruiz et al. have presented an optimization procedure based on simulated annealing which has been performed to enhance the design of micro-perforated panels, so that the absorptive capabilities of those panels can be fine-tuned to an optimal [6]. In micro-perforated panels, the absorption phenomena do not only rely on dissipative mechanisms (friction losses within micro-perforations), but also on resonant properties (coupling with a back-cavity for example). Through optimization study, an optimal setting has been achieved, and such methodology is demonstrated to allow fine-tuning of the absorbing capabilities with respect to the frequency range of interest. 
In general, modifying a factor (or parameter) at a time often leads to select a wrong optimum, due to potential interactions between factors which are not taken into account. To alleviate this situation, the response surface methodology (RSM) has been applied to optimize the acoustic performances of an electroacoustic absorber, since such devices are intrinsically multivariate systems. This method was first introduced by Box and Wilson [7] for developing empirical models of complex processes that could be used to locally represent a process response [7]. A review of the literature of RSM including theoretical aspects and practical applications were carried out by Hill and Hunter [8]. Basically, RSM is used for replacing an overall process by an approximate model based on a series of results collected at various discrete points within the design space. Low-order polynomial functions (second-order is often implemented) are generally employed as they can efficiently model low-order processes, since the processing of the corresponding response surface is fast and cheap. The most extensive applications of RSM can be found in the realm of industrial engineering, particularly in situations where numerous explanatory variables can potentially influence a performance measure, or quality characteristic, of products or processes. The efficiency of the RSM as an advantageous optimization method is documented in many fields and a number of improvements of the method is presented in the literature [7-11].

\section{Electroacoustic absorbers}

\subsection{General presentation}

An electroacoustic absorber is an electroacoustic loudspeaker system, including enclosure and acoustic circuit, the acoustic impedance of which can be varied by various electrical means, be it passive or active. In the specific case of the electrodynamic moving-coil loudspeaker given in Fig. 1, the lumped elements model of such devices includes a moving mass $M_{m s}$, a mechanical compliance $C_{m s}$, a mechanical resistance $R_{m s}$, as well as coupling factors (force factor $B l$, and radiating surface $S$ ). The above-mentioned elements also account for the mechanical counterparts of the acoustic radiation impedances (for instance $C_{a b}=V_{b} / \rho c^{2}$ the acoustic compliance of the enclosure of volume $V_{b}$, where $\rho$ is the density of air and $c$ the celerity of sound in air, or the acoustic radiation mass and resistance). At last, the electrical conditioning of the loudspeaker electrical terminals is also accounted as equivalent mechanical elements, including the d.c. resistance and self induc-

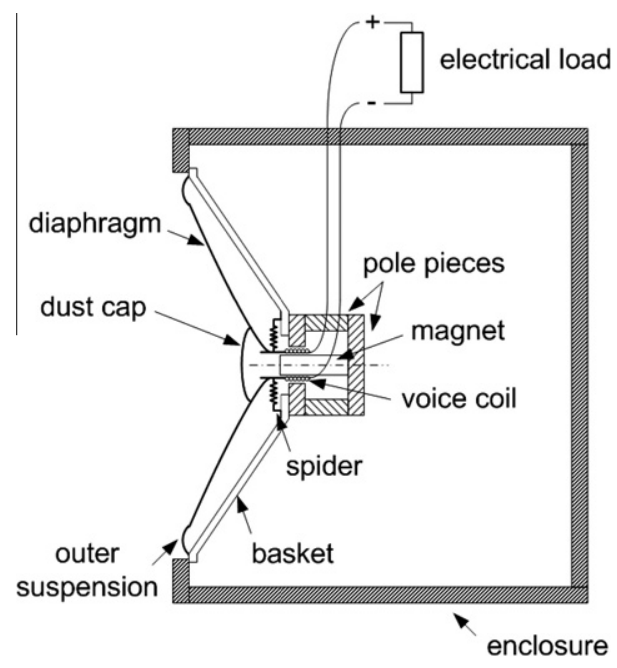

Fig. 1. Schematic of electroacoustic absorber. tance of the coil $R_{e}$ and $L_{e}$, but also any electric load that shunts the loudspeaker electrical terminals [12]. If the electric load is a simple resistor $R_{S}$ (passive shunt), it has been proven that such device can generally be characterized in terms of equivalent normalized admittance, the expression of which is of the form [1]:

$Y=Z_{m c} \frac{1}{R_{m s}+\frac{B l^{2}}{R_{e}+R_{s}}+j \omega M_{m s}+\frac{1}{j \omega C_{m s}}}$

where $Z_{m c}=S Z_{c}$ is the equivalent mechanical impedance of characteristic impedance of air, namely $Z_{c}=\rho c$. For electroacoustic absorbers, the targeted objective functions is usually a global measure of sound energy absorption, namely the acoustic absorption coefficient $\alpha$ :

$\alpha=1-\left|\frac{1-Y}{1+Y}\right|^{2}$

To provide a complete absorption, the coefficient must equal 1 . If it is not, the performances of the absorber can be increased through optimization design, which is one of the main motivation of this paper.

\subsection{Acoustic performance assessment}

Measurement of sound absorption coefficient under normal incidence of actual electroacoustic absorbers can be performed in an impedance tube configuration, after ISO 10534-2 standard, using the two-microphone transfer function method [13]. The corresponding one-dimensional experimental setup is described in Fig. 2. Using this setup, the acoustic absorption coefficient is derived from the assessment of sound pressure at two different positions in an impedance tube, one extremity of which is closed with the electroacoustic absorber. The formulation of the absorption coefficient is given below:

$\alpha=1-|r|^{2}$

where $r$ is the reflection coefficient, processed after:

$r=\frac{H_{12}-H_{I}}{H_{R}-H_{12}} \exp \left(2 j k x_{1}\right)$

The term $H_{12}=p_{1} / p_{2}$ is the transfer function between the two sound pressure $p_{1}$ and $p_{2}$ sensed at positions 1 and 2 (see Fig. 2), $H_{I}=\exp \left(-j k\left(x_{1}-x_{2}\right)\right)$ and $H_{R}=\exp \left(j k\left(x_{1}-x_{2}\right)\right)$ are the transfer functions corresponding to the incident $p_{i}$ and reflected $p_{r}$ sound waves respectively, $x_{1}$ being the position of the most distant microphone from the absorber under study and $k$ the wave number. Alternatively, the resulting acoustic performances of electroacoustic absorbers can be assessed in the context of actual rooms, especially in the low-frequency range, and more precisely below the Schroeder frequency. In a recent publication [4] dealing with modal control in rooms, 10 electroacoustic absorbers distributed in a line array configuration and placed at several positions in a reverberant chamber, each single absorber being primarily tuned in an impedance tube, have been assessed and demonstrated to be equally effective for damping several modes at a time in the room, the damping capabilities being more effective within the band (20$40 \mathrm{~Hz}$ ). In the following, we will then focus on optimizing and assessing single electroacoustic absorbers in a one-dimensional configuration, assuming those performances can be easily extrapolated to practical 3D configurations.

\section{Response surface methodology}

The methodology of response surface (RSM) is an experimental strategy for exploring the space of a process involving a number of explanatory variables by using empirical statistical models. Using 


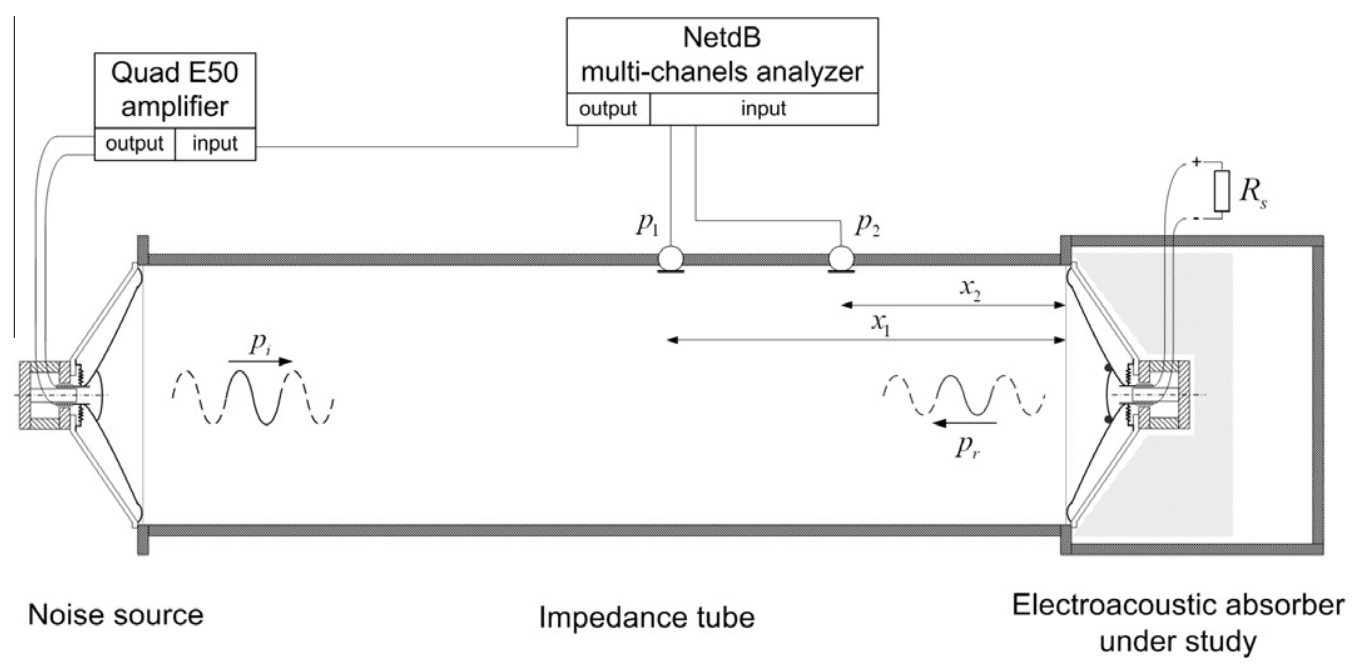

Fig. 2. Experimental setup for the assessment of electroacoustic absorber's absorption coefficient under normal incidence.

this approach, a complex process is replaced by an approximate model based on experimental data evenly collected within the space of the process by varying simultaneously some influential parameters in a structured way. It also includes an optimization method for finding the levels of the controllable input variables that produce desirable values of the response.

\subsection{Objective function estimation}

The RSM strategy aims at developing an appropriate approximated relationship between obtained responses and explanatory variables. To establish this matching, polynomial functions of second-order are frequently used. The general form of response surface models of second-order with interactions that describes the relationship between the response variable of interest $y$ and $m$ independent explanatory variables may be written as:

$y=b_{0}+\sum_{i=1}^{m} b_{i} x_{i}+\sum_{i=1, j>i}^{m} b_{i j} x_{i} x_{j}+\sum_{i=1}^{m} b_{i i} x_{i}^{2}+\varepsilon$

where $y$ approximates the objective function, $x_{i}$ and $x_{j}$ are the independent explanatory variables, $b_{i}$ and $b_{i j}$ represent the polynomial coefficients to identify, and $\varepsilon$ the error associated to $y$, that represents other not accounted sources of variability which are assumed to be normally distributed. Starting from the general form of Eq. (5), the polynomial function restricted to four explanatory variables can be expressed as follows:

$$
\begin{aligned}
y= & b_{0}+b_{1} x_{1}+b_{2} x_{2}+b_{3} x_{3}+b_{4} x_{4}+b_{12} x_{1} x_{2}+b_{13} x_{1} x_{3} \\
& +b_{14} x_{1} x_{4}+b_{23} x_{2} x_{3}+b_{24} x_{2} x_{4}+b_{34} x_{3} x_{4}+b_{11} x_{1}^{2}+b_{22} x_{2}^{2} \\
& +b_{33} x_{3}^{2}+b_{44} x_{4}^{2}+\varepsilon
\end{aligned}
$$

For $n$ observations, the model of Eq. (6) may be written in matrix form as:

$\boldsymbol{y}=\boldsymbol{X} \beta+\varepsilon$

where $\boldsymbol{y}$ is an $n \times 1$ vector of the observations, $\boldsymbol{X}$ is an $n \times p$ design matrix, $\beta$ is a $p \times 1$ vector of the regression coefficients or effects, and $\varepsilon$ is a $n \times 1$ vector of random error or noise. The method of least mean squares is commonly used to estimate the unknown regression coefficients in a multiple regression analysis. At last the unknown $\beta$ terms can be obtained from the formula:

$\beta=\left(\boldsymbol{X}^{\prime} \boldsymbol{X}\right)^{-1} \boldsymbol{X}^{\prime} \boldsymbol{y}$
The $\beta$ vector is composed with the unknown parameters set which can be estimated by collecting experimental data. The collected data can either be derived from actual physical experiments or from numerical models of the same experiments. When the $\beta$ terms are substituted into the second-order response surface model given in Eq. (6), the approximating polynomial function can be predicted at any explanatory variable $x_{i}$. Once a response surface model is obtained, statistical analysis techniques are usually processed to check the fitness of the mathematical model, and then a canonical analysis can be performed to investigate the shape of the predicted response surface.

\subsection{Decomposition of variance}

The key to determining the overall utility of the regression equation lies in assessing its ability to account for the variance observed in the response variable. The objective of variance analysis is to estimate to what extent the whole model and its individual parameters contribute to an understanding of the response variable under study. In other words, this test procedure aims at describing if changes in the response are caused by changes in the action between different levels, or by random fluctuations due to the dispersion of responses. The required theory of variance analysis is listed in Table 1 .

In this table, the total sum of squares $S S_{T}$ measures the overall fluctuations of the individual observations of the dependant variables around their average. The regression sum of squares $S S_{R}$ is the sum of the squared differences between the values of the dependent variable predicted by the regression line $\hat{y}_{i}$ and those predicted by the mean $\bar{y}$. The residual sum of squares $S S_{E}$ represents the sum of the squared differences between the observed values $y_{i}$ of the response and the ones predicted by the regression $\hat{y}_{i}$. To estimate whether certain actions of the model are significant or not, we use the experimental and critical tabulated values of Fisher $F$-test. Referring to probability statistics, $F=M S_{R} / M S_{E}$ is the ratio of the explained variability and the unexplained variability, each divided by the corresponding degrees of freedom [14,15]. The larger the F-test, the more useful the model. When the level of significance $\sigma$ is specified, the critical value $F_{p, n-p-1, \sigma}$ that satisfies the probability:

$P\left\{F>F_{p, n-p-1, \sigma}\right\}=\sigma$

can be looked up from $F$-distribution tables. If $F>F_{p, n-p-1, \sigma}$, then the second-degree polynomial can be considered as a reliable model at 
Table 1

Variance analysis table.

\begin{tabular}{|c|c|c|c|c|c|}
\hline & Sum of squares & Degree of freedom & Mean squares & $F$-test & $p$-value \\
\hline Regression & $S S_{R}=\Sigma\left(\hat{y}_{i}-\bar{y}\right)^{2}$ & $p$ & $M S_{R}=S S_{R} / p$ & $F=\frac{M S_{R}}{M S_{E}}$ & $F>F_{p, n-p-1, \sigma}$ \\
\hline Error & $S S_{E}=\Sigma\left(y_{i}-\bar{y}\right)^{2}$ & $n-p-1$ & $M S_{E}=S S_{E} /(n-p-1)$ & & \\
\hline Total & $S S_{T}=\Sigma\left(y_{i}-\bar{y}\right)^{2}$ & $n-1$ & & & \\
\hline
\end{tabular}

Table 2

Loudspeaker Visaton AL-170 technical data.

\begin{tabular}{ll} 
Nominal impedance & $8 \Omega$ \\
Resonance frequency & $38 \mathrm{HZ}$ \\
Moving mass $M_{m s}$ & $13 \mathrm{~g}$ \\
d.c resistance $R_{e}$ & $5.6 \Omega$ \\
Inductance of voice coil $L_{e}$ & $0.9 \mathrm{mH}$ \\
Force factor $B l$ & $6.9 \mathrm{Tm}$ \\
Effective piston area $S$ & $133 \mathrm{~cm}^{2}$ \\
Equivalent volume $V_{a s}$ & 341 \\
Mechanical $Q$ factor $Q_{m s}$ & 3.88 \\
Electrical $Q$ factor $Q_{e s}$ & 0.43 \\
Total $Q$ factor $Q_{t s}$ & 0.39 \\
\hline
\end{tabular}

$\sigma$ level. This test procedure is therefore a mean to improve the regression equation.

\subsection{Canonical analysis}

The main reason for performing a canonical analysis is to gain insight into the nature of the response surface, i.e. if the response is a maximum, a minimum, or a saddle point. Moreover, the effects of input variables combinations which have not been carried out in the designed experiments can still be considered [10]. The quadratic fitted model which relates independent explanatory variables $\boldsymbol{x}=\left(x_{1}, x_{2}, \ldots, x_{m}\right)$ to a response variable $y$ (see Eq. (6)) can also be expressed in matrix notation as:

$\hat{\boldsymbol{y}}=b_{0}+\boldsymbol{x} \boldsymbol{b}+\boldsymbol{x}^{\prime} \widehat{\boldsymbol{B}} \boldsymbol{x}$

where $b_{0}, \boldsymbol{b}$, and $\widehat{\boldsymbol{B}}$ are the estimates of the constant, linear, and second-order coefficients, respectively.

$\begin{aligned} \widehat{\boldsymbol{B}} & =\frac{1}{2}\left(\begin{array}{llll}2 b_{11} & b_{12} & \ldots & b_{1 m} \\ b_{12} & 2 b_{22} & \ldots & \cdots \\ \ldots & \cdots & 2 b_{33} & \ldots \\ b_{1 m} & \cdots & \ldots & 2 b_{m m}\end{array}\right) \\ \boldsymbol{b} & =\left(\begin{array}{c}b_{1} \\ b_{2} \\ \ldots \\ b_{m}\end{array}\right)\end{aligned}$

$\boldsymbol{x}=\left(\begin{array}{llll}x_{1} & x_{2} & \ldots & x_{m}\end{array}\right)$

To find the best estimates for the parameters $\widehat{\boldsymbol{B}}, \boldsymbol{b}$, and $b_{0}$, statistically designed experiments are employed. Once a model has been estimated for a particular space of a process, the direction of maximum gradient is found by normalizing the factors and differentiating on $\boldsymbol{x}$.

$\frac{\partial \hat{\boldsymbol{y}}}{\partial \boldsymbol{x}}=\boldsymbol{b}+2 \widehat{\boldsymbol{B}} \boldsymbol{x}$

Setting the second term of Eq. (14) to 0 yields the location of the stationary point $\boldsymbol{x}_{s}$ and the predicted response $\boldsymbol{y}_{s}[10]$ : $\boldsymbol{x}_{s}=-\frac{1}{2} \widehat{\boldsymbol{B}}^{-1} \boldsymbol{b}$

$\boldsymbol{y}_{s}=b_{0}+\frac{1}{2} \boldsymbol{x}_{s}^{\prime} \boldsymbol{b}$

The nature of the stationary point is determined with the signs of the eigenvalues of matrix $\widehat{B}$, and the relative magnitude of eigenvalues are also helpful in the total interpretation of the response system. At last, the canonical equation for the response surface is expressed as:

$\hat{\boldsymbol{y}}=\boldsymbol{y}_{s}+\sum_{i=1}^{f} \lambda_{i} w_{i}^{2}$

where $\lambda_{i}$ is the eigenvalue of matrix $\widehat{\boldsymbol{B}}$ associated to the explanatory variable $x_{i}$, and $w_{i}$ are called the canonical variables.

\section{Designed experiments of electroacoustic absorbers}

\subsection{Design factors and variation ranges}

The design factors are the explanatory variables over which the experiments can be actually controlled. In the frame of this study the selected factors are some constitutive parameters of an electroacoustic absorber which reflect some dissipative mechanisms of sound energy, and are also controllable. The corresponding variables are given in Fig. 3. The variation ranges are the physical constraints of each factors, or the limitations imposed by the experimental setup, that define and limit the space of the process.

The selected design factors, whose subscripts from Eq. (1) are removed for ease of writing, are listed hereafter:

- The moving mass $M$ of the moving-coil loudspeaker.

- The enclosure volume $V$ of the closed-box.

- The filling density of mineral fiber $\tau$.

- The electrical load value $R$ to which the loudspeaker is connected.

As presented earlier, the sound absorption coefficient $\alpha$ which is measured in one-third octave bands is used as response variable depending on the frequency.

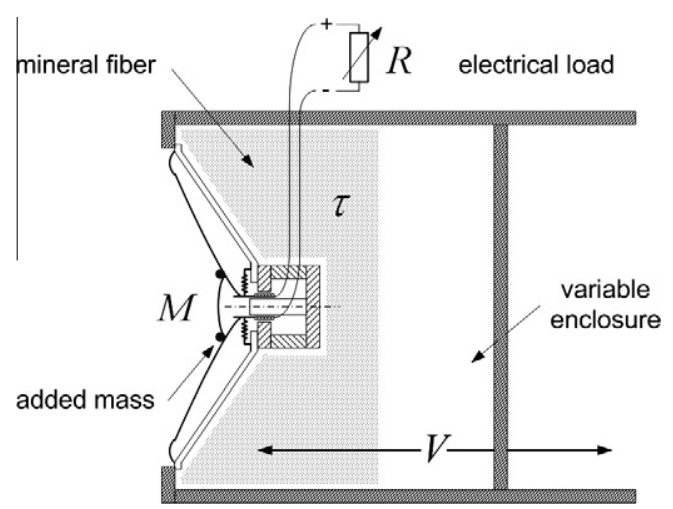

Fig. 3. Schematic of electroacoustic absorber including design factors. 
To improve the understanding of the phenomenon by allowing heterogeneous comparison of quantities, it is convenient to transform natural variables into coded variables. Natural variables are expressed in physical units of measurement, whereas coded variables are dimensionless, have zero mean and the same standard deviation. The applied transformations are given hereafter:

$$
\begin{array}{ll}
x_{1}=\frac{M-\bar{M}}{\Delta M} ; & x_{2}=\frac{V-\bar{V}}{\Delta V} \\
x_{3}=\frac{\tau-\bar{\tau}}{\Delta \tau} ; & x_{4}=\frac{\log R-\log \bar{R}}{\log \Delta R}
\end{array}
$$

where for the case of design factor $M, \bar{M}=\left(M_{\min }+M_{\max }\right) / 2$ and $\Delta M=\left(M_{\max }-M_{\min }\right) / 4$, the same applying for the other design factors $V, \tau$, and $R$. As it can be seen in Eq. (18), a logarithmic transformation has been chosen to take account of the large range that relates to the electrical load factor. Using this transformation, physical values of design factors are transformed into coded values -2 , $-1,0,1,2$. Table 3 shows the corresponding values between both natural and coded levels.

In order to practically increase the moving mass of the loudspeaker, a certain quantity of sinkers has been stuck to the diaphragm, as illustrated in Fig. 4. Moreover, in order to allow variation of the enclosure volume, a specific cabinet has been designed which was filled with a variable quantity of mineral fiber according the specifications of the designed experiments (see Fig. 4).

Table 3

Coded and natural levels of design factors.

\begin{tabular}{llrlrr}
\hline Coded levels & -2 & -1 & 0 & 1 & 2 \\
\hline Natural levels & & & & & \\
Moving mass $\left(10^{-3} \mathrm{~kg}\right)$ & 13 & 17 & 21 & 25 & 29 \\
Enclosure volume $\left(10^{-3} \mathrm{~m}^{3}\right)$ & 4 & 12 & 20 & 28 & 36 \\
Filling density $(\%)$ & 0 & 25 & 50 & 75 & 100 \\
Electrical load $(\Omega)$ & 0.1 & 1 & 10 & 100 & 1000 \\
\hline
\end{tabular}

\subsection{Designed experiments for fitting response surfaces}

In statistics, factorial experiments are a class of designed experiments for which the explanatory variables are varied simultaneously, but in a structured way. Such a design consists of two or more factors, each with discrete possible values or levels. The experiments can take all possible combinations of the levels of each factor. However, two-levels factorial designs are insufficient to fit a second-order model. Indeed, with two-levels factorial designs, each factor is only investigated at an upper and a lower level, which bounds the space of the process. Such a design requires the assumption of no curvature within the design space, whereas to describe an extremum, one must estimate quadratic curvature requiring at least three levels for each factor. In order to attain the optimum response, three-levels factorial designs were developed by Box and Behnken [9]. As this study focuses on fitting the second-order model given in Eq. (6) which contains $p=15$ parameters to be estimated, at least 15 different combinations of design factors must be estimated. However, such designs with $m$ input variables involve a great number of runs $n=3^{m}$ compared to the $p$ coefficients to be determined.

To alleviate this situation, central composite designs (CCD) have been developed. This class of experimental designs involves the use of a two-levels factorial, one combined with a $2 \mathrm{~m}$ axial or star points and $n_{0}$ center runs [10]. Center runs clearly provide information about the existence of curvature in the system or process under study. As the former are often replicated this can also estimate experimental error. If curvature is found, the addition of axial points allows for estimation of the pure quadratic terms. The value of the axial distance generally varies from 1 to $\sqrt{m}$ which guarantees that the CCD is effective from a variance point of view. The number of runs to be made in both orthogonal or rotatable CCD are thus $n=2^{m}+2 m+n_{0}$, sensibly lower than tree-levels factorial designs. For these reasons, CCD are popular for fitting a second-order surface in experimental optimization processes. In Table 4, the conducted central composite design is illustrated with coded vari-
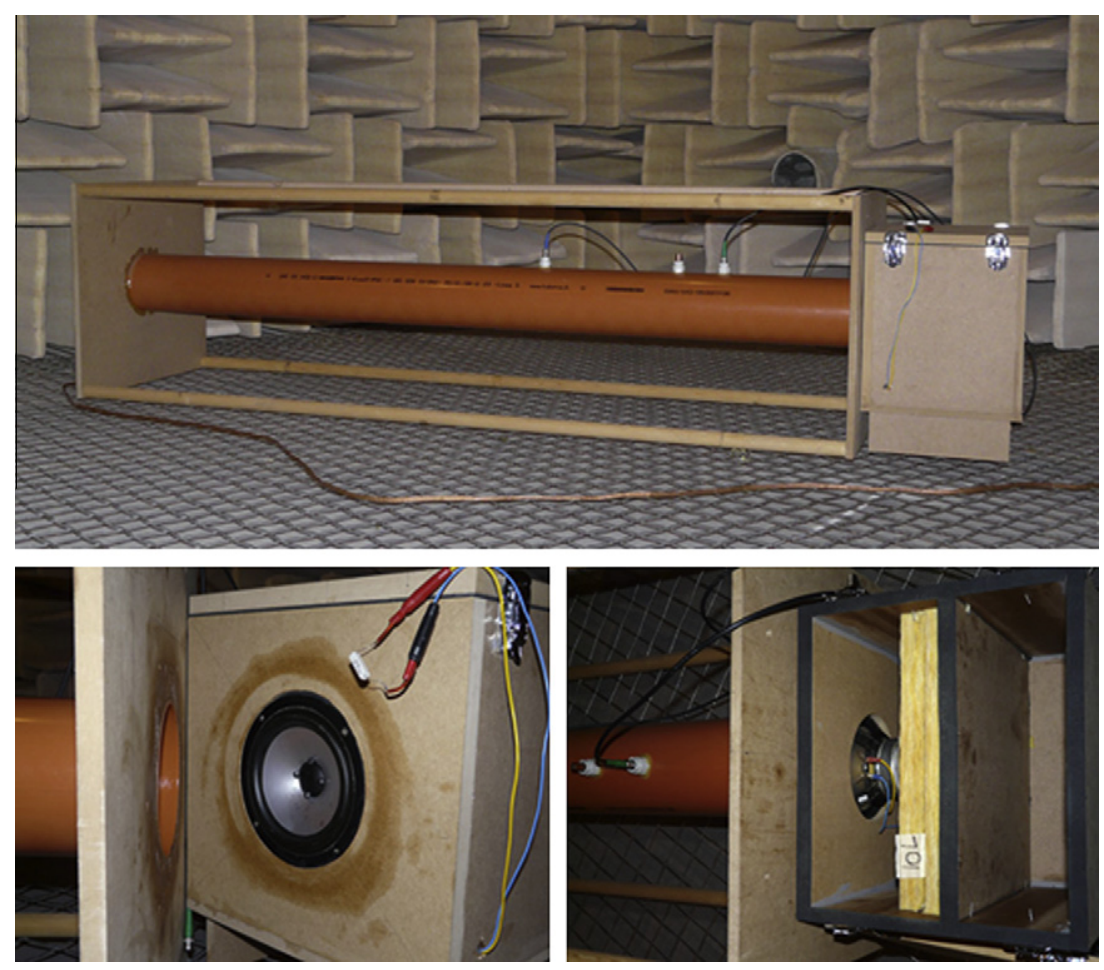

Fig. 4. Overview of the experimental setup. 
Table 4

Central composite design in coded variables.

\begin{tabular}{|c|c|c|c|c|}
\hline \multirow[t]{2}{*}{ Run \# } & \multicolumn{4}{|c|}{ Design matrix } \\
\hline & $x_{1}$ & $x_{2}$ & $x_{3}$ & $x_{4}$ \\
\hline \multicolumn{5}{|c|}{ Full factorial design } \\
\hline 4 & -1 & -1 & -1 & -1 \\
\hline 20 & -1 & -1 & -1 & 1 \\
\hline 12 & -1 & -1 & 1 & -1 \\
\hline 6 & -1 & -1 & 1 & 1 \\
\hline 8 & -1 & 1 & -1 & -1 \\
\hline 17 & -1 & 1 & -1 & 1 \\
\hline 25 & -1 & 1 & 1 & -1 \\
\hline 10 & -1 & 1 & 1 & 1 \\
\hline 13 & 1 & -1 & -1 & -1 \\
\hline 21 & 1 & -1 & -1 & 1 \\
\hline 11 & 1 & -1 & 1 & -1 \\
\hline 5 & 1 & -1 & 1 & 1 \\
\hline 18 & 1 & 1 & -1 & -1 \\
\hline 19 & 1 & 1 & -1 & 1 \\
\hline 26 & 1 & 1 & 1 & -1 \\
\hline 9 & 1 & 1 & 1 & 1 \\
\hline \multicolumn{5}{|c|}{ Axial points } \\
\hline 2 & -2 & 0 & 0 & 0 \\
\hline 23 & 2 & 0 & 0 & 0 \\
\hline 30 & 0 & -2 & 0 & 0 \\
\hline 29 & 0 & 2 & 0 & 0 \\
\hline 3 & 0 & 0 & -2 & 0 \\
\hline 15 & 0 & 0 & 2 & 0 \\
\hline 24 & 0 & 0 & 0 & -2 \\
\hline 28 & 0 & 0 & 0 & 2 \\
\hline \multicolumn{5}{|c|}{ Center runs } \\
\hline 1 & 0 & 0 & 0 & 0 \\
\hline 7 & 0 & 0 & 0 & 0 \\
\hline 14 & 0 & 0 & 0 & 0 \\
\hline 16 & 0 & 0 & 0 & 0 \\
\hline 22 & 0 & 0 & 0 & 0 \\
\hline 27 & 0 & 0 & 0 & 0 \\
\hline
\end{tabular}

ables. The description on curve fitting by multiple linear regression, the corresponding analysis of variance and the way for finding the optimum response can be found in Refs. $[10,16]$.

\section{Results and discussion}

\subsection{Measured responses}

Figs. 5-8 illustrate the curves of the one-third octave bands absorption coefficients which were measured randomly during the experiment. Coming back to our problem about low-frequency noise control in rooms, some specifications have to be drawn for the electroacoustic absorber in terms of sound absorption performances. In view of damping the first modes in the control room of a recording studio, which dimension are $3.40 \mathrm{~m} \times$ $2.10 \mathrm{~m} \times 2.15 \mathrm{~m}$ the electroacoustic absorber is primarily intended to dissipate sound energy around $50 \mathrm{~Hz}$. Therefore, the following analysis will be focused on the normal $50 \mathrm{~Hz}$ one-third octave band. Table 5 summarizes both measured and estimated responses (namely the acoustic absorption coefficient) for the one-third octave band $50 \mathrm{~Hz}$. The physical levels of the natural variables are detailed for each of the $n=30$ experimental runs carried out randomly.

\subsection{Analysis of variance}

Starting from the experimental results shown in Table 5, an analysis of the variance is performed to investigate the validity of the regression model. Table 6 illustrates the result of the ANOVA which was carried out for a level of significance of $5 \%$, i.e., for a $95 \%$ level of confidence. The last column of the table shows the

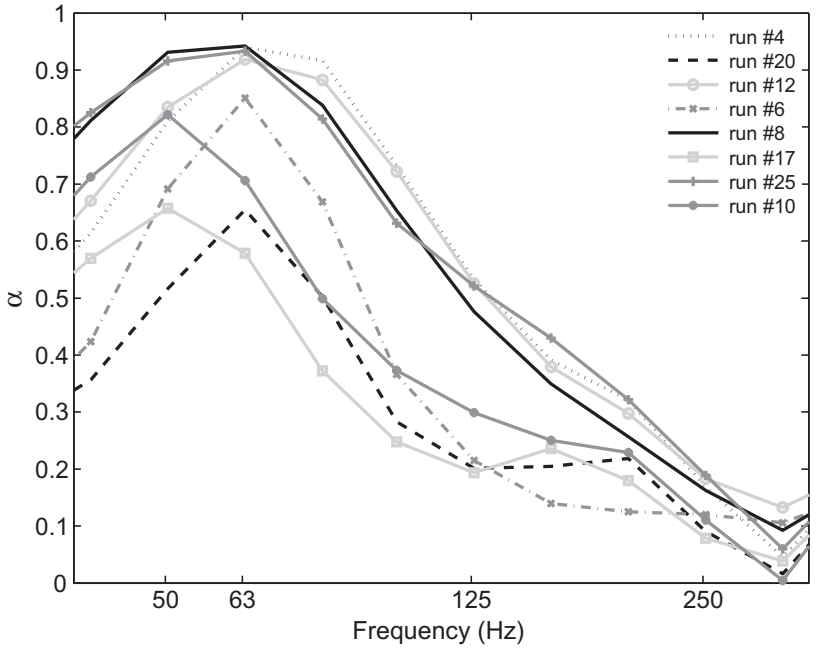

Fig. 5. Measured absorption coefficients of the factorial design.

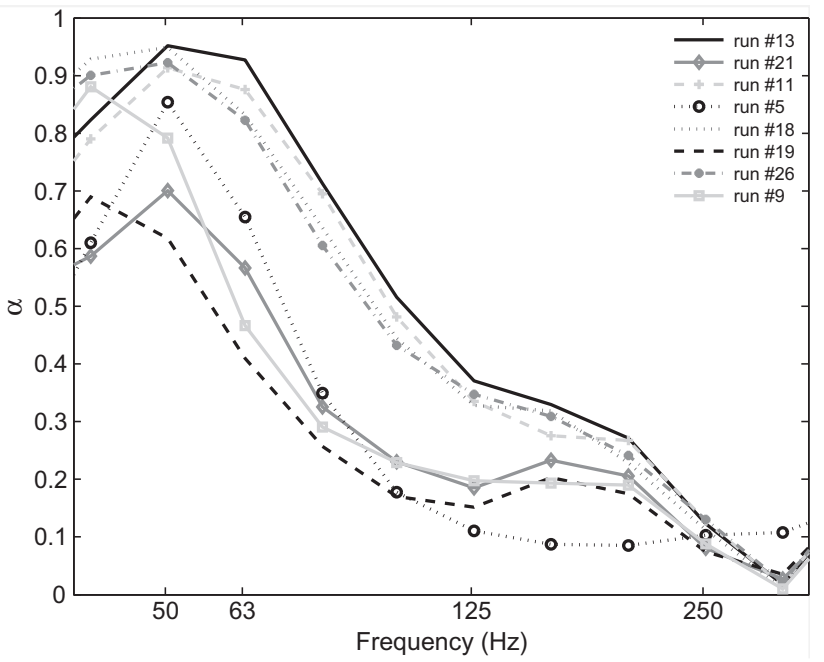

Fig. 6. Measured absorption coefficients of the factorial design.

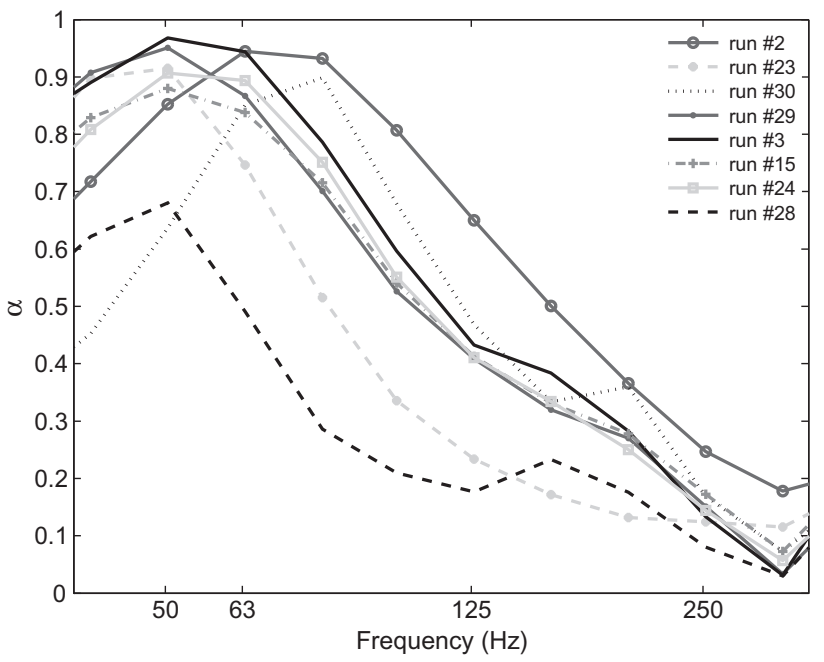

Fig. 7. Measured absorption coefficients at axial points of design space. 


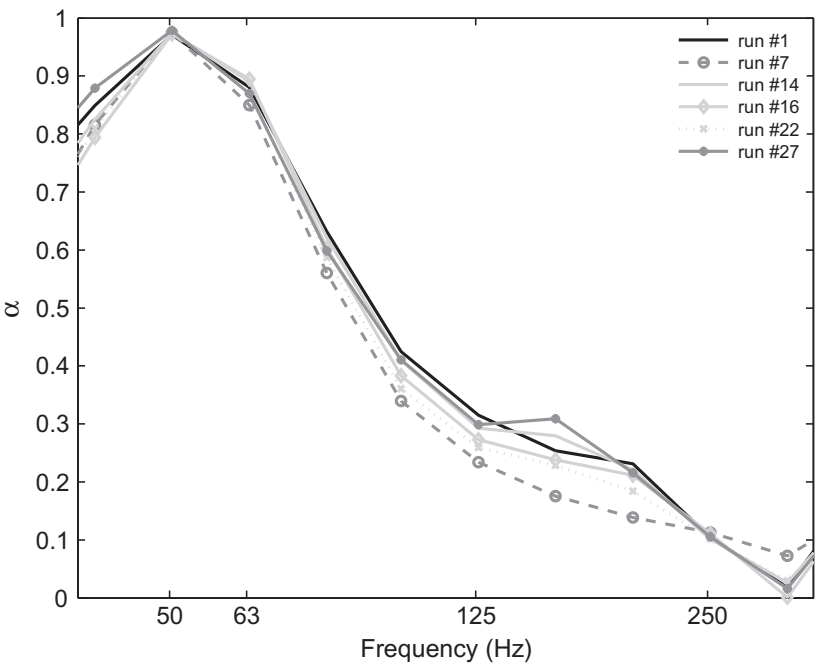

Fig. 8. Measured absorption coefficients at the center of design space.

Table 5

Measured and estimated responses.

\begin{tabular}{|c|c|c|c|c|c|c|}
\hline \multirow[t]{2}{*}{ Run \# } & \multicolumn{3}{|c|}{ Experimental design } & \multicolumn{3}{|c|}{ Responses } \\
\hline & $M\left(10^{-3} \mathrm{~kg}\right)$ & $V\left(10^{-3} \mathrm{~m}^{3}\right)$ & $\tau(\%)$ & $R(\Omega)$ & $y$ & $\bar{y}$ \\
\hline 4 & 17 & 12 & 25 & 1 & 0.81 & 0.84 \\
\hline 20 & 17 & 12 & 25 & 100 & 0.52 & 0.58 \\
\hline 12 & 17 & 12 & 75 & 1 & 0.83 & 0.78 \\
\hline 6 & 17 & 12 & 75 & 100 & 0.69 & 0.70 \\
\hline 8 & 17 & 28 & 25 & 1 & 0.93 & 0.99 \\
\hline 17 & 17 & 28 & 25 & 100 & 0.66 & 0.74 \\
\hline 25 & 17 & 28 & 75 & 1 & 0.92 & 0.94 \\
\hline 10 & 17 & 28 & 75 & 100 & 0.82 & 0.86 \\
\hline 13 & 25 & 12 & 25 & 1 & 0.95 & 0.97 \\
\hline 21 & 25 & 12 & 25 & 100 & 0.70 & 0.71 \\
\hline 11 & 25 & 12 & 75 & 1 & 0.91 & 0.91 \\
\hline 5 & 25 & 12 & 75 & 100 & 0.85 & 0.81 \\
\hline 18 & 25 & 28 & 25 & 1 & 0.95 & 0.97 \\
\hline 19 & 25 & 28 & 25 & 100 & 0.62 & 0.72 \\
\hline 26 & 25 & 28 & 75 & 1 & 0.92 & 0.92 \\
\hline 9 & 25 & 28 & 75 & 100 & 0.79 & 0.84 \\
\hline 2 & 13 & 20 & 50 & 10 & 0.85 & 0.87 \\
\hline 23 & 29 & 20 & 50 & 10 & 0.92 & 0.98 \\
\hline 30 & 21 & 4 & 50 & 10 & 0.64 & 0.66 \\
\hline 29 & 21 & 36 & 50 & 10 & 0.95 & 0.82 \\
\hline 3 & 21 & 20 & 0 & 10 & 0.97 & 0.89 \\
\hline 15 & 21 & 20 & 100 & 10 & 0.88 & 0.96 \\
\hline 24 & 21 & 20 & 50 & 0.1 & 0.91 & 0.91 \\
\hline 28 & 21 & 20 & 50 & 1000 & 0.68 & 0.57 \\
\hline 1 & 21 & 20 & 50 & 10 & 0.97 & 0.93 \\
\hline 7 & 21 & 20 & 50 & 10 & 0.97 & 0.93 \\
\hline 14 & 21 & 20 & 50 & 10 & 0.97 & 0.93 \\
\hline 16 & 21 & 20 & 50 & 10 & 0.97 & 0.93 \\
\hline 22 & 21 & 20 & 50 & 10 & 0.98 & 0.93 \\
\hline 27 & 21 & 20 & 50 & 10 & 0.98 & 0.93 \\
\hline
\end{tabular}

Table 6

Variance analysis and evaluation of the regression model.

\begin{tabular}{lrllll}
\hline & \multicolumn{1}{c}{$S S$} & $d f$ & $M S$ & $F$-test & $p$-value \\
\hline Second-order & & & & & \\
Regression & 22.12 & 15 & 1.5 & 370 & $<0.001$ \\
Error & 0.06 & 14 & 0.004 & & \\
Total & 22.18 & 29 & & & \\
\hline
\end{tabular}

Table 7

ANOVA table for each individual effects.

\begin{tabular}{lrlrrr}
\hline Source & \multicolumn{1}{l}{ SS } & $d f$ & \multicolumn{1}{l}{$M S$} & F-test & $p$-value \\
\hline Main affects & & & & & \\
$M$ & 0.018 & 1 & 0.018 & 4.41 & 0.053 \\
$V$ & 0.039 & 1 & 0.039 & 9.82 & 0.007 \\
$\tau$ & 0.007 & 1 & 0.070 & 1.75 & 0.205 \\
$R$ & 0.172 & 1 & 0.172 & 43.02 & $<0.001$ \\
Interaction effects & & & & & \\
$M \times V$ & 0.023 & 1 & 0.023 & 5.83 & 0.029 \\
$M \times \tau$ & 0.001 & 1 & 0.001 & 0.13 & 0.727 \\
$M \times R$ & $<0.001$ & 1 & $<0.001$ & 0.01 & 0.907 \\
$V \times \tau$ & $<0.001$ & 1 & $<0.001$ & 0.01 & 0.969 \\
$V \times R$ & 0.001 & 1 & 0.001 & 0.01 & 0.727 \\
$\tau \times R$ & 0.032 & 1 & 0.032 & 7.89 & 0.013 \\
Quadratic effects & & & & & \\
$M \times M$ & 0.007 & 1 & 0.007 & 1.82 & 0.197 \\
$V \times V$ & 0.053 & 1 & 0.053 & 13.30 & 0.002 \\
$\tau \times \tau$ & 0.004 & 1 & 0.004 & 1.05 & 0.322 \\
$R \times R$ & 0.075 & 1 & 0.075 & 18.71 & 0.001 \\
Error & 0.060 & 14 & 0.004 & & \\
Total & 22.183 & 29 & & & \\
\hline
\end{tabular}

contribution ( $p$-value) of the second-order regression model on the total variation.

From the analysis of Table 6, it is obvious that the F-test of ANOVA is greater than the critical value. Indeed, by using F-distribution tables we obtain $F_{(15,14,0.05)}=2.46, F=370>F_{(15,14,0.05)}$ for the second-order regression model. The polynomial representing absorption coefficient is therefore relevant for the corresponding problem. It can be used to analyze the relationship between the objective performance function and the selected constitutive parameters $(M, V, \tau$, and $R)$ of the electroacoustic absorber. The remaining step consists now in investigating the significance of each factorial effect within the global regression model.

Table 7 summarizes the result of the test for significance using ANOVA with a level of significance of $5 \%$ for each individual regressor coefficients of the model.

From the analysis of Table 7, the F-test of some effects appears to be lower than $F_{(1,14,0.05)}=4.60$. We conclude that the quadratic terms $M \times M$ and $\tau \times \tau$, and interaction terms $M \times \tau, M \times R, V \times \tau$, and $V \times R$ have statistically insignificant effect. It can be also noted that, within the frequency range of interest, namely the $50 \mathrm{~Hz}$ onethird octave band, the most important effects are influenced by the electrical load value and the enclosure volume. This complementary ANOVA indicates that some terms of Eq. (6) might be removed to get a better model. By removing statistically nonsignificant effects from the second-order model, we obtain a reduced model (Eq. (19)) whose ANOVA is given in Table 8.

$$
\begin{aligned}
y= & b_{0}+b_{1} x_{1}+b_{2} x_{2}+b_{3} x_{3}+b_{4} x_{4}+b_{12} x_{1} x_{2}+b_{34} x_{3} x_{4} \\
& +b_{22} x_{2}^{2}+b_{44} x_{4}^{2}
\end{aligned}
$$

From the analysis of Table 8, it is apparent that the F-test of ANOVA $F=540$ is greater than the critical value $F_{(9,20,0.05)}=2.39$ Therefore, we conclude that the reduced regression model is relevant and can be used in the following.

Table 8

Variance analysis of reduced regression model.

\begin{tabular}{lcrlll}
\hline & \multicolumn{1}{c}{ SS } & $d f$ & $M S$ & $F$-test & $p$-value \\
\hline Second-order & & & & & \\
Regression & 22.12 & 9 & 2.46 & 540 & $<0.001$ \\
Error & 0.092 & 20 & 0.0046 & & \\
Total & 22.18 & 29 & & & \\
\hline
\end{tabular}




\subsection{Fitted second-order response function}

The coefficients of the reduced model are estimated by the least mean squares method, as detailed in Section 3. The fitted secondorder response function which is obtained after regression is given in coded variables by:

$$
\begin{aligned}
\hat{y}= & 0.924+0.027 x_{1}+0.040 x_{2}+0.017 x_{3}-0.085 x_{4} \\
& -0.038 x_{1} x_{2}+0.044 x_{3} x_{4}-0.046 x_{2}^{2}-0.046 x_{4}^{2}
\end{aligned}
$$

In natural variables Eq. (20) becomes:

$$
\begin{aligned}
\hat{\alpha}= & -0.011+30.5 M+58.7 V-0.11 \tau-0.08 \log (R) \\
& -1187.5 M V+0.18 \tau \log (R)-718.8 V^{2}-0.05 \log (R)^{2}
\end{aligned}
$$

A similar approach can be envisaged by considering values measured in other range of frequency. The resulting models would be simply different from those obtained in Eqs. (20) and (21).

\subsection{Canonical analysis}

After the polynomial has been verified, the RSM is processed in Matlab ${ }^{\infty}$. From Eq. (15), the stationary point is observed at $\boldsymbol{x}_{s}=(-0.65,0.71,1.12,-0.38)$, and the predicted value is $\boldsymbol{y}_{s}=0.96$ according to Eq. (16). It can be noted that the distance between $\boldsymbol{x}_{s}$ and the center of the design space is lower than the radius of the design space, meaning that the stationary point is included within the experimental domain of investigations. If the point was located outside the region of the experiment, it would not be advisable to use it for defining operating conditions because the fitted model is only reliable inside the design space.

The computed eigenvalues of matrix $\widehat{\boldsymbol{B}}$ are $\lambda_{1}=-0.055$, $\lambda_{2}=-0.053, \lambda_{3}=0.007$, and $\lambda_{4}=0.009$. The canonical form is therefore given by:

$$
\hat{y}=0.96-0.055 w_{1}^{2}-0.053 w_{2}^{2}+0.007 w_{3}^{2}+0.009 w_{4}^{2}
$$

As the eigenvalues are mixed in sign, the stationary point $\boldsymbol{x}_{S}$ is a saddle point. Eq. (22) represents a minimax surface where a decrease in yield is predicted when one moves away from the center of the system in either the positive or negative directions of $w_{1}$ and $w_{2}$, and correspondingly an increase in yield is predicted in either the positive or negative directions of $w_{3}$ and $w_{4}$. In Eq. (22) it can be observed that all eigenvalues are not of the same order of magnitude. This means that the same displacement in each of the main directions do not cause a comparable variation of the response. Canonical analysis through eigenvalues of matrix $\widehat{\boldsymbol{B}}$ yields to the main directions which tend to increase (or decrease) the response faster, and to what extent. Response surfaces and the contour plots can then be employed in order to find optimum conditions and to determine more precisely how sensitive the estimated response is for any displacement away from the stationary point.

\subsection{Estimation of optimum configurations through response surfaces and contour plots}

It is often necessary for practical reason to use constrained optimization to identify potential operating conditions. This is particularly true when the stationary point is a saddle point. The response surface and contour plots provide then one of the most revealing ways of illustrating and interpreting the responses surfaces system. Such graphical displays derived from the polynomial model of process under study, after fixing some factors so as to estimate the response while other factors are free to vary. For ease of graphical representation, only two factors are free to vary while the two other ones are held constant. The following sections illustrate various configurations obtained from Eq. (21) in order to estimate the levels of factors which yield an optimum.

\subsubsection{Moving mass vs. enclosure volume}

The response surfaces and contour plots in Figs. 9 and 10 show the effect of the moving mass $M$ and enclosure volume $V$ on the sound absorption coefficient under the condition that the filling density of mineral fiber $\tau$ and electrical load value $R$ are held constant. When the enclosure is partially filled with mineral fiber materials $(\tau=75 \%$ ), and when an electrical load $R=1 \Omega$ is connected to the electric terminals of the loudspeaker, two areas of optimum conditions can be identified. Indeed, the figures show that a full absorption may be expected for the natural levels $\left(M \approx 13 \times 10^{-3} \mathrm{~kg}\right.$ and $\left.V \approx 28 \times 10^{-3} \mathrm{~m}^{3}\right)$, as well as for $(M>$ $25 \times 10^{-3} \mathrm{~kg}$ and $V \approx 20 \times 10^{-3} \mathrm{~m}^{3}$ ). When the volume of the enclosure is left empty ( $\tau=0 \%$ ) and the electroacoustic absorber

a
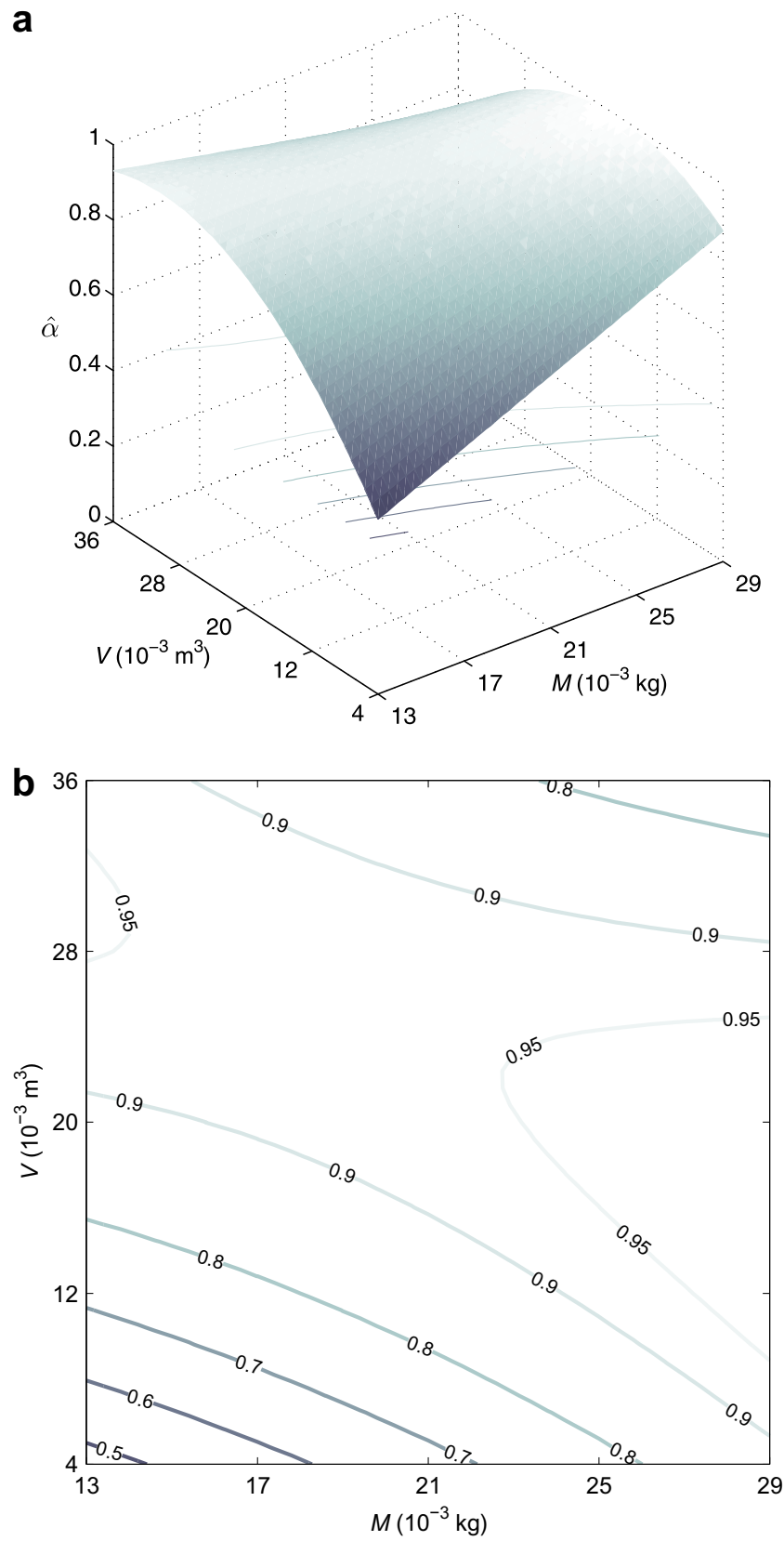

Fig. 9. Moving mass vs. volume, $\tau=75 \%$ and $R=1 \Omega$. 
a
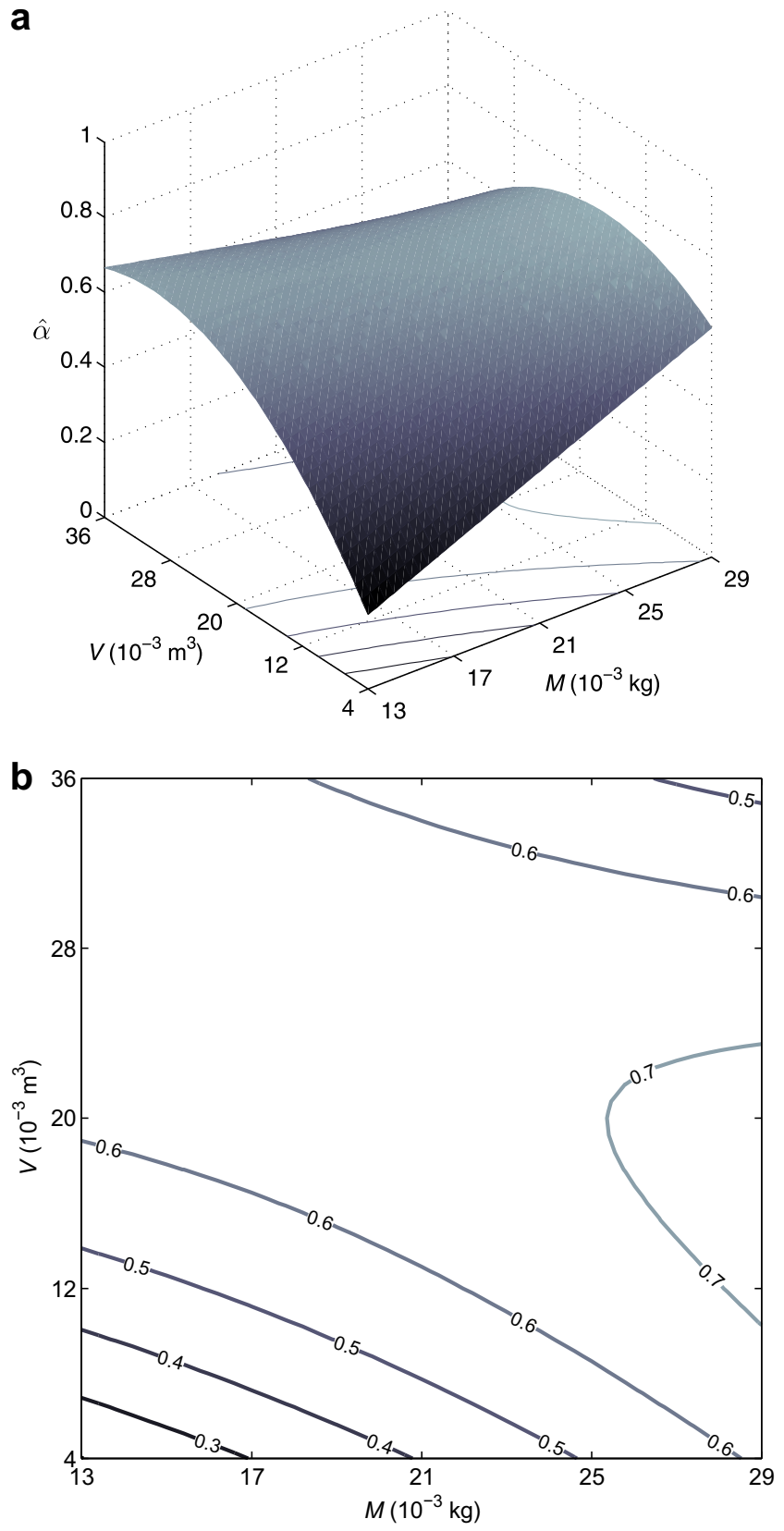

Fig. 10. Moving mass vs. volume, $\tau=0 \%$ and $R=100 \Omega$.

is shunted with a high electrical load value $(R=100 \Omega)$, one can observe that the optimum conditions are drastically different. With such adjustment, it is not expected to have good absorbent properties, anywhere in the design space (see Fig. 10).

\subsubsection{Moving mass vs. electrical load}

Figs. 11 and 12 illustrate the effect of $M$ and $R$ over the sound absorption coefficient under the condition that $\tau$ and $V$ are held constant. In the case of a volume of $12 \times 10^{-3} \mathrm{~m}^{3}$ which is partially filled with mineral fiber ( $\tau=25 \%$ ), we can clearly identify the optimum operating conditions around $\left(M>25 \times 10^{-3} \mathrm{~kg}\right.$ and $\left.R \approx 1 \Omega\right)$. For other levels of $V$ and $\tau$, the expected response is slightly different. For a larger volume of the enclosure and without any mineral fiber, the expected optimum conditions depend mainly on $R$, and on $M$ to a worse extent (see Fig. 12). a
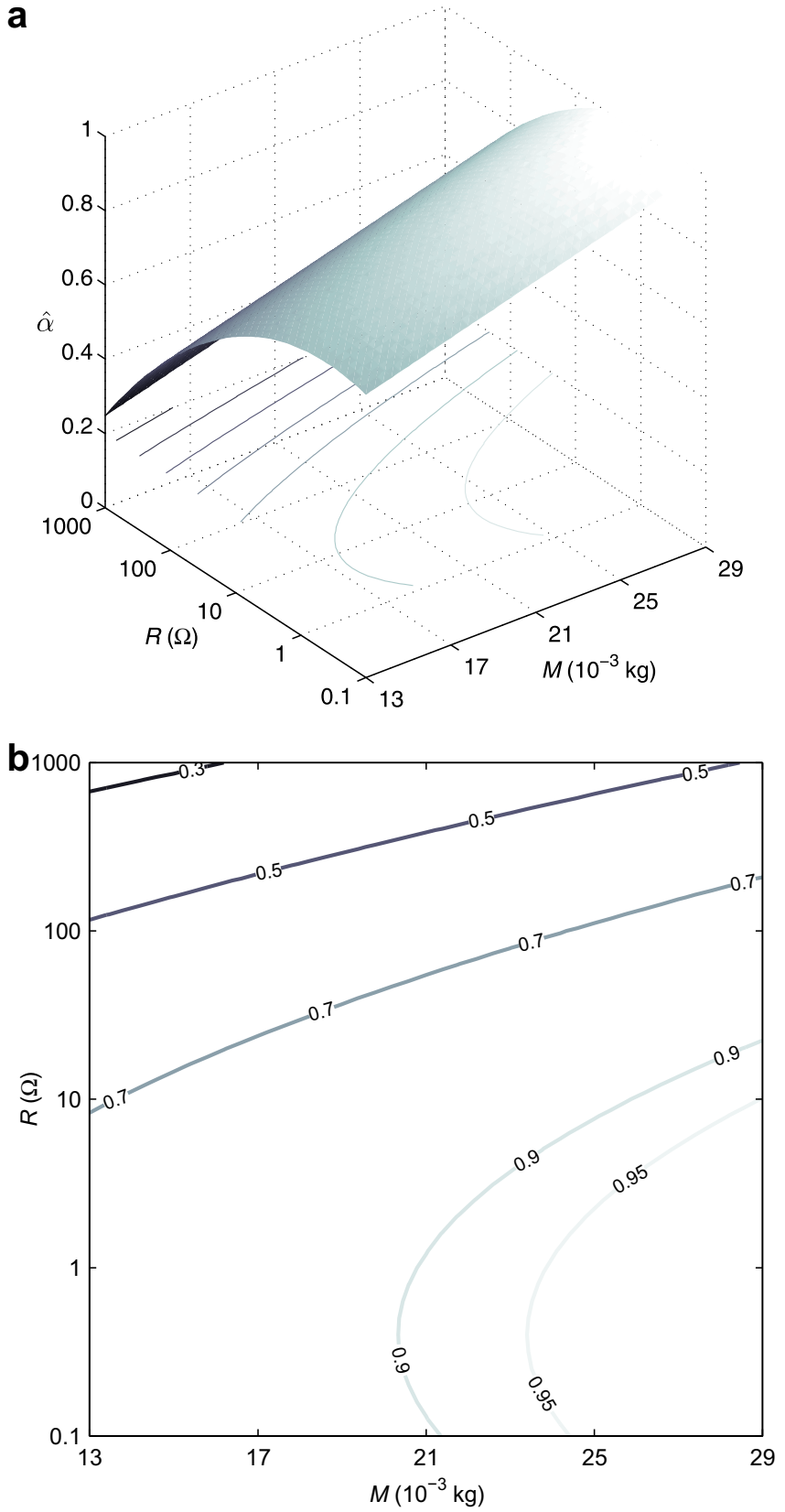

Fig. 11. Moving mass vs. electrical load, $\tau=25 \%$ and $V=12 \times 10^{-3} \mathrm{~m}^{3}$.

\subsubsection{Enclosure volume vs. electrical load}

Figs. 13 and 14 illustrate the effect of $V$ and $R$ over the sound absorption coefficient under the condition that $\tau$ and $M$ are held constant. For a moving mass $M=21 \times 10^{-3} \mathrm{~kg}$ and without any mineral fiber within the enclosure, we identify the optimum conditions when $V \approx 24 \times 10^{-3} \mathrm{~m}^{-3}$ and $R \approx 1 \Omega$. When no mass is added to the loudspeaker's moving mass $\left(M=13 \times 10^{-3} \mathrm{~kg}\right)$, and when the enclosure is partially filled with mineral fiber $(\tau=25 \%)$, the trend is nearly the same (see Fig. 14).

\subsubsection{Enclosure volume vs. filling density}

Figs. 15 and 16 illustrate the effect of $V$ and $\tau$ on the sound absorption coefficient under the condition that $R$ and $M$ are held constant. For a moving mass $M=21 \times 10^{-3} \mathrm{~kg}$ and an electrical load of $1 \Omega$, the volume of the enclosure needs to be close to $24 \times 10^{-3} \mathrm{~m}^{-3}$ so as to attain optimum conditions. However, the 
a
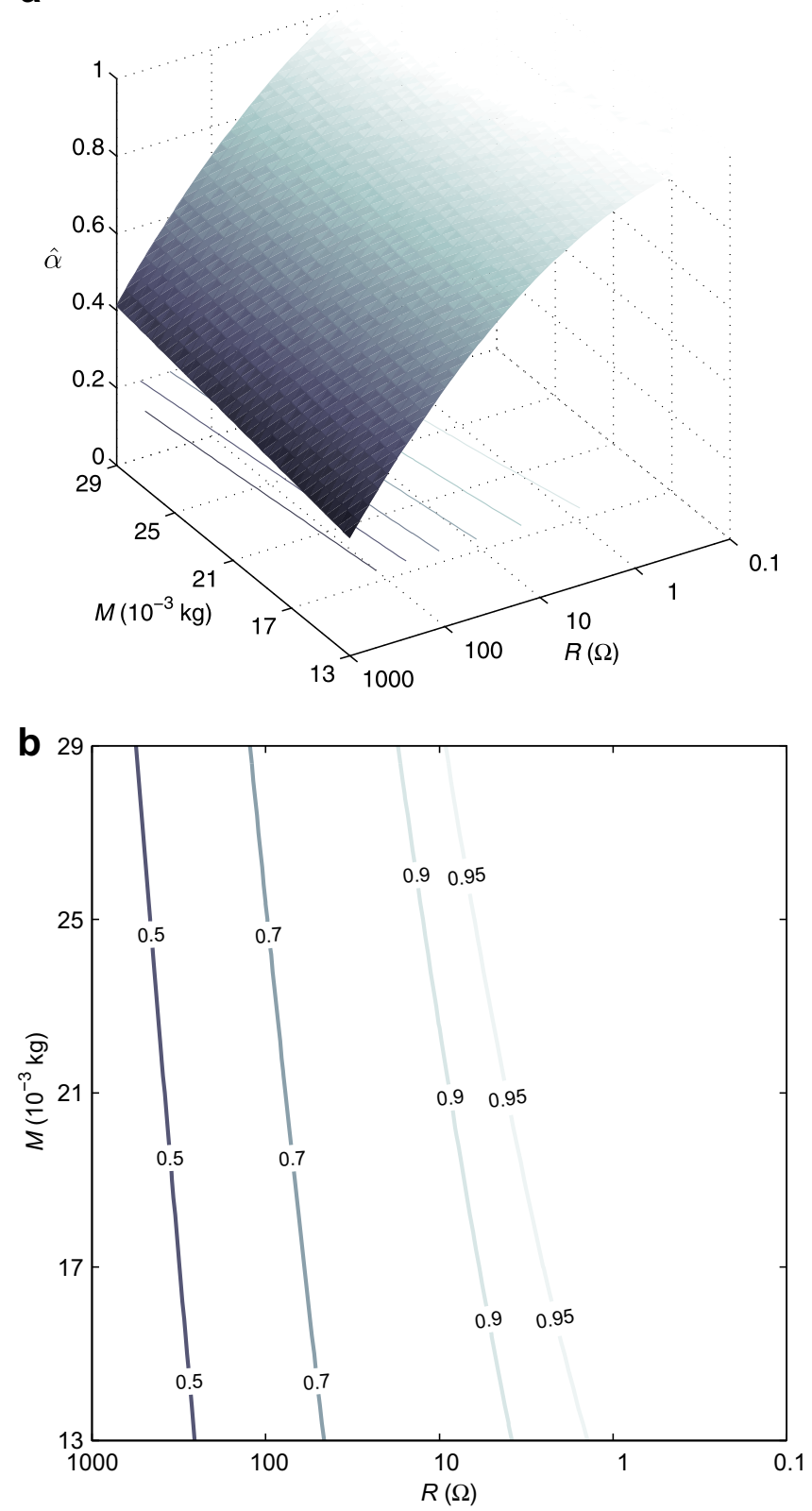

Fig. 12. Moving mass vs. electrical load, $\tau=0 \%$ and $V=20 \times 10^{-3} \mathrm{~m}^{3}$.

requirement for $\tau$ is less drastic, as illustrated in the Fig. 16. When the moving mass is smaller and the electrical load higher, the volume should be slightly larger $\left(V \approx 28 \times 10^{-3} \mathrm{~m}^{3}\right)$ and the filling density upper than $50 \%$. With such an adjustment however, the expected acoustic performance of the absorber should be worse.

\subsection{Constraints imposed by the room modal control application}

Since the electroacoustic absorber has been designed for a modal control application in rooms, especially the first specific modes around $50 \mathrm{~Hz}$, the main design constraints are relative to the size of the device, and hence the volume of the enclosure to be embedded into walls. The results that follow include two configurations with a fixed volume of $10 \mathrm{l}$ initially left empty and then partially filled with mineral fiber. The objective is to find optimum operating conditions associated for both cases. For a volume left empty, a
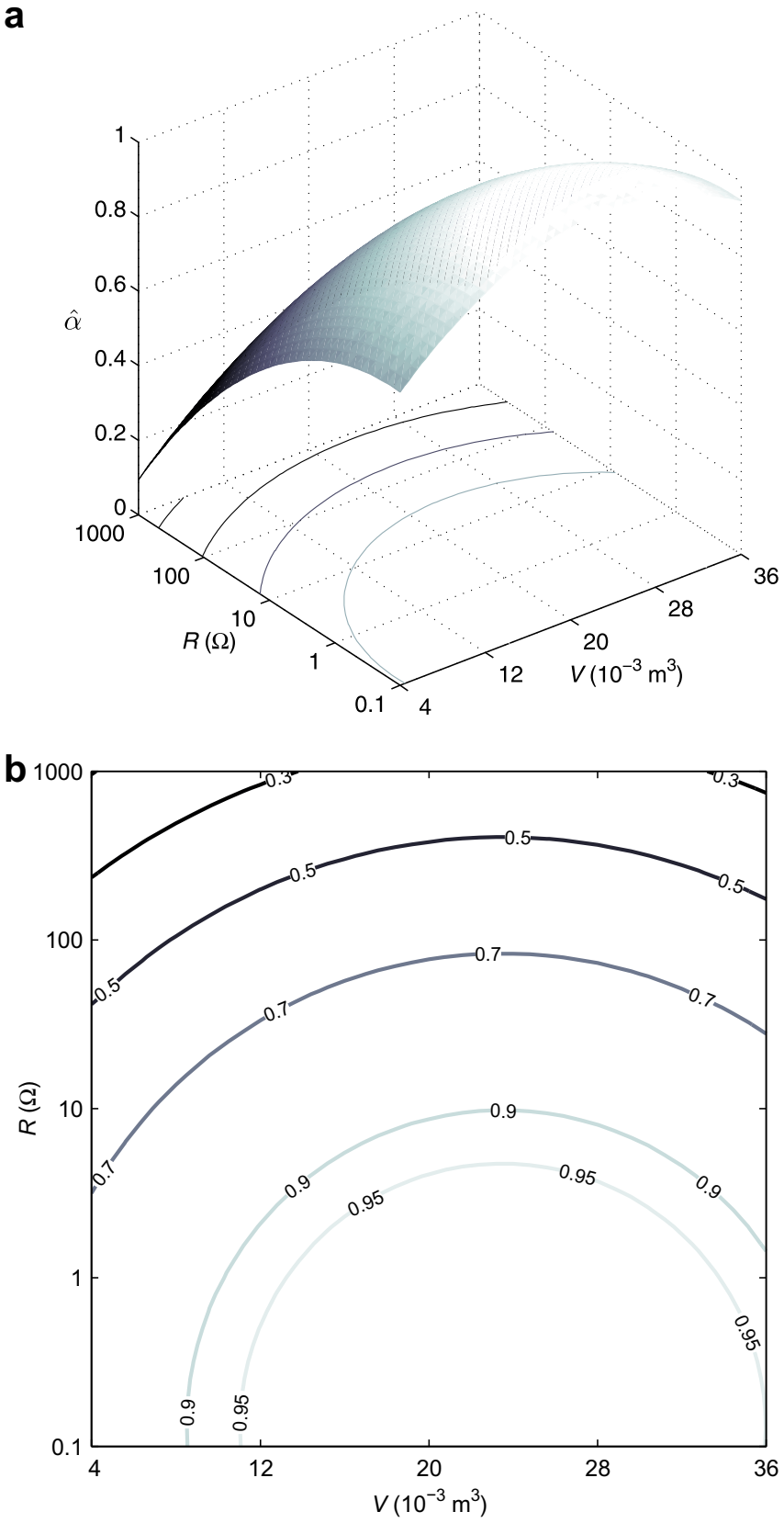

Fig. 13. Volume vs. electrical load, $\tau=0 \%$ and $M=21 \times 10^{-3} \mathrm{~kg}$.

i.e. with $\tau=0 \%$, the expected behavior of the electroacoustic absorber is illustrated in Fig. 17.

For a volume partially filled with mineral fiber, i.e. with $\tau=80 \%$, the predicted absorption coefficient within the bounds of explanatory variables $R$ and $M$ is illustrated in Fig. 18.

These contour plots will help us identify the optimum operating conditions within the imposed constraints. By tuning the electroacoustic absorber at the levels marked by a cross on the contour plots, Eq. (21) estimates the two following absorption coefficients for the $50 \mathrm{~Hz}$ one-third octave band:

$\hat{\alpha}(M=0.027, V=0.01, \tau=0, R=0.68) \approx 1$

$\hat{\alpha}(M=0.029, V=0.01, \tau=0.8, R=10) \approx 0.97$

In order to validate those computed responses, measurements were performed at these levels after ISO-10534-2 standard using the same setup as depicted in Fig. 2. The two measured curves 
a
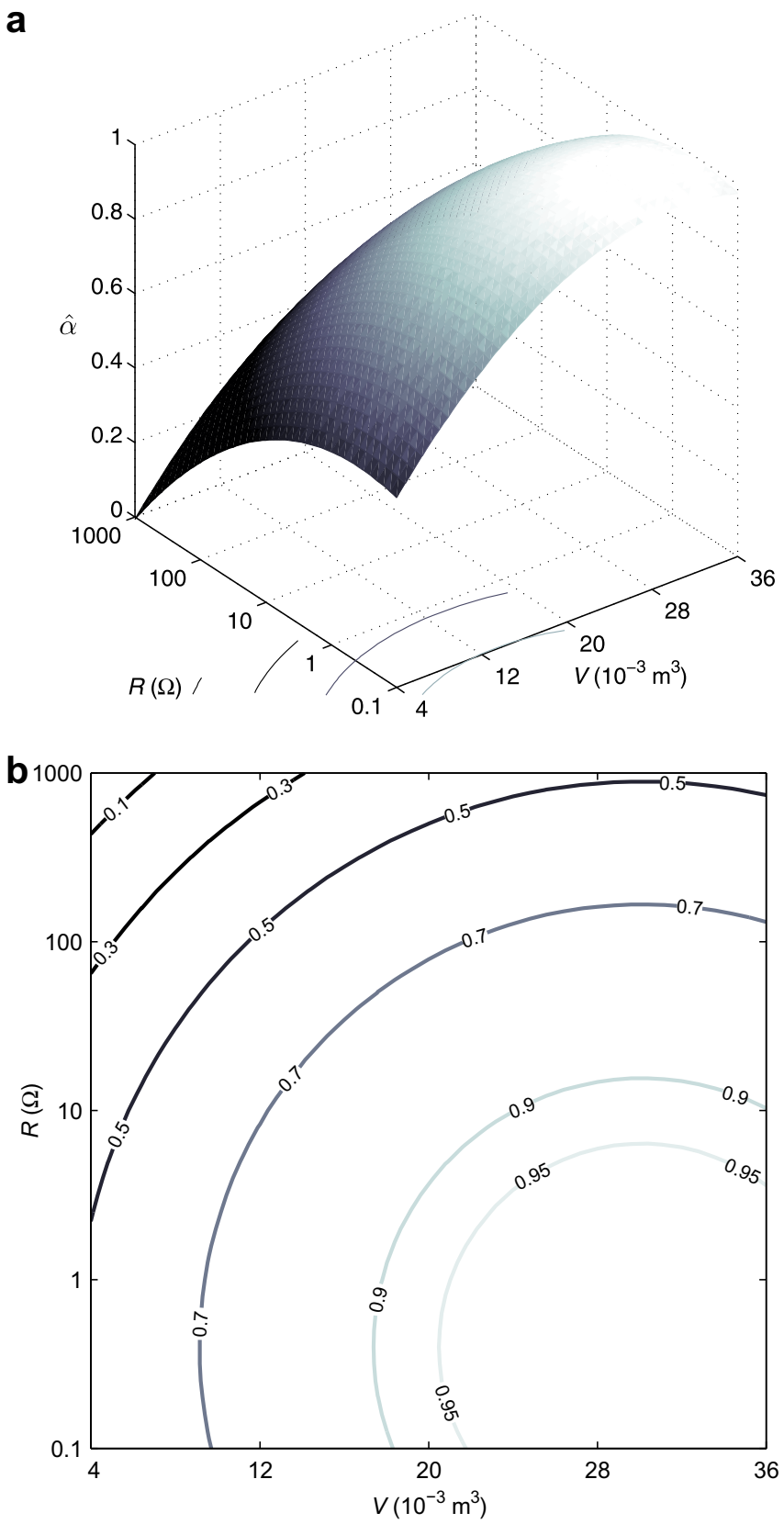

Fig. 14. Volume vs. electrical load, $\tau=25 \%$ and $M=13 \times 10^{-3} \mathrm{~kg}$.

representing the one-third octave bands absorption coefficient for the configurations given by Eqs. (23) and (24) are presented in Fig. 19.

Fig. 19 clearly shows that computed values which are obtained from the fitted second-order response function match the measured absorption coefficient in both cases. Moreover, it can be noted that the required value of electrical load to which the loudspeaker is connected depends on the filling density of mineral fiber. For an enclosure left empty, the optimum operating conditions are expected for a value of electrical load lower than the d.c. resistance of the moving-coil loudspeaker (see Table 2). Conversely, for an enclosure partially filled with mineral fiber, the value of electrical load needs to be upper the d.c. resistance.

In order to assess the capabilities of such devices to damp several modes at a time, the electroacoustic absorber has been installed at one end of a $4 \mathrm{~m}$ length duct with the objective to attenuate the first resonant mode around $49 \mathrm{~Hz}$. A main sound a
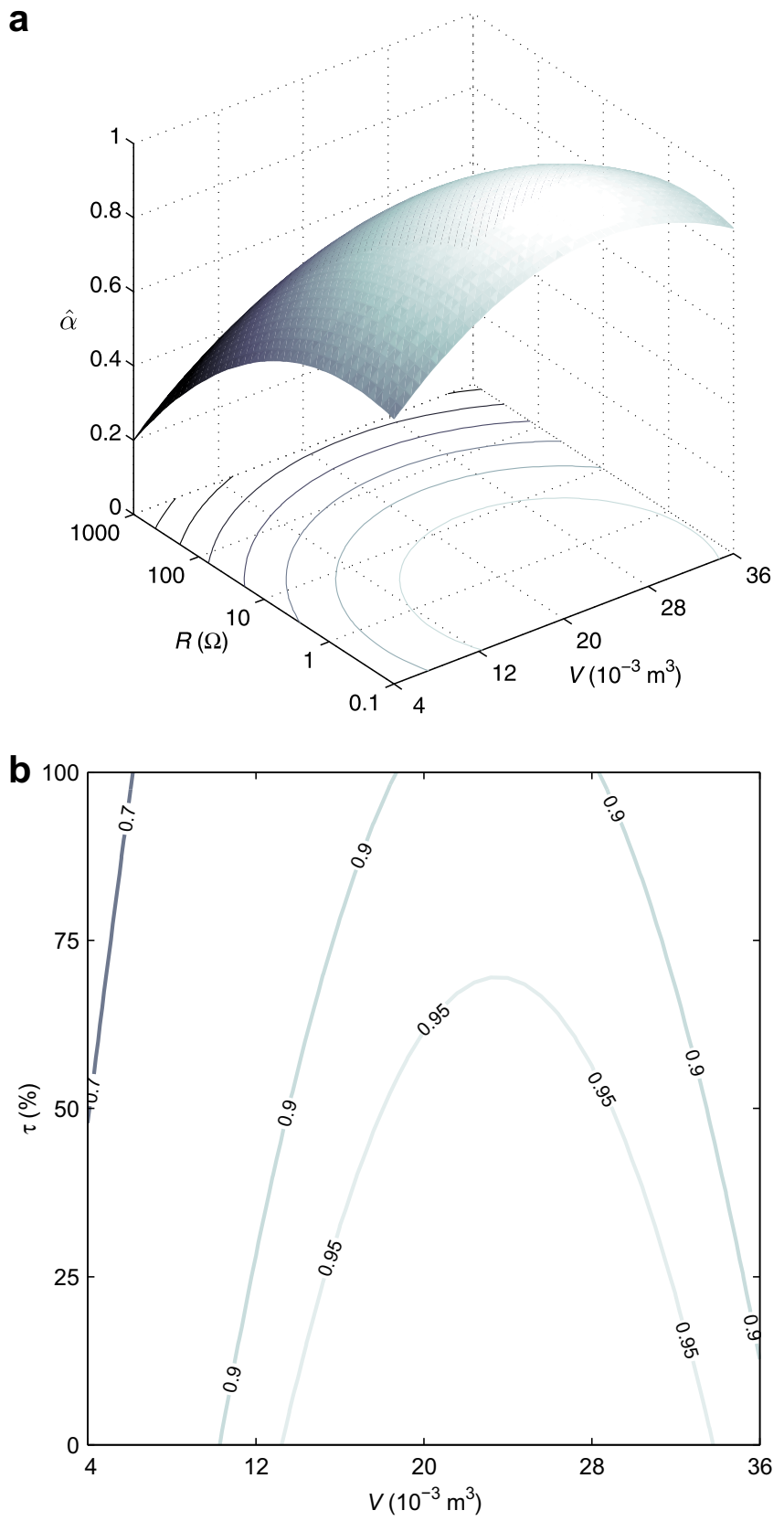

Fig. 15. Filling density vs. volume, $R=1 \Omega$ and $M=21 \times 10^{-3} \mathrm{~kg}$.

source delivering a swept sine excitation is placed at the opposite end and the resulting sound pressure level is measured with a microphone close to the electroacoustic absorber. Fig. 20 show the measured sound pressure level in case of hard wall and with the electroacoustic absorber in the configurations given by Eqs. (23) and (24).

Fig. 20 clearly shows that the electroacoustic absorber after optimization for the $50 \mathrm{~Hz}$ one-third octave band yields a firstmode attenuation of more than $12 \mathrm{~dB}$. Compared to the hard wall configuration, that is to say when the opposite end of the sound source is rigid, it can be observed that the electroacoustic absorber does not affect the response for higher frequencies but tends to slightly attenuate the second resonant mode of more than $4 \mathrm{~dB}$. It is important to note that only a few modes close to the resonant frequency of the loudspeaker can be controlled. Thus, depending on the frequency range of interest, the selection of a suitable loud- 
a
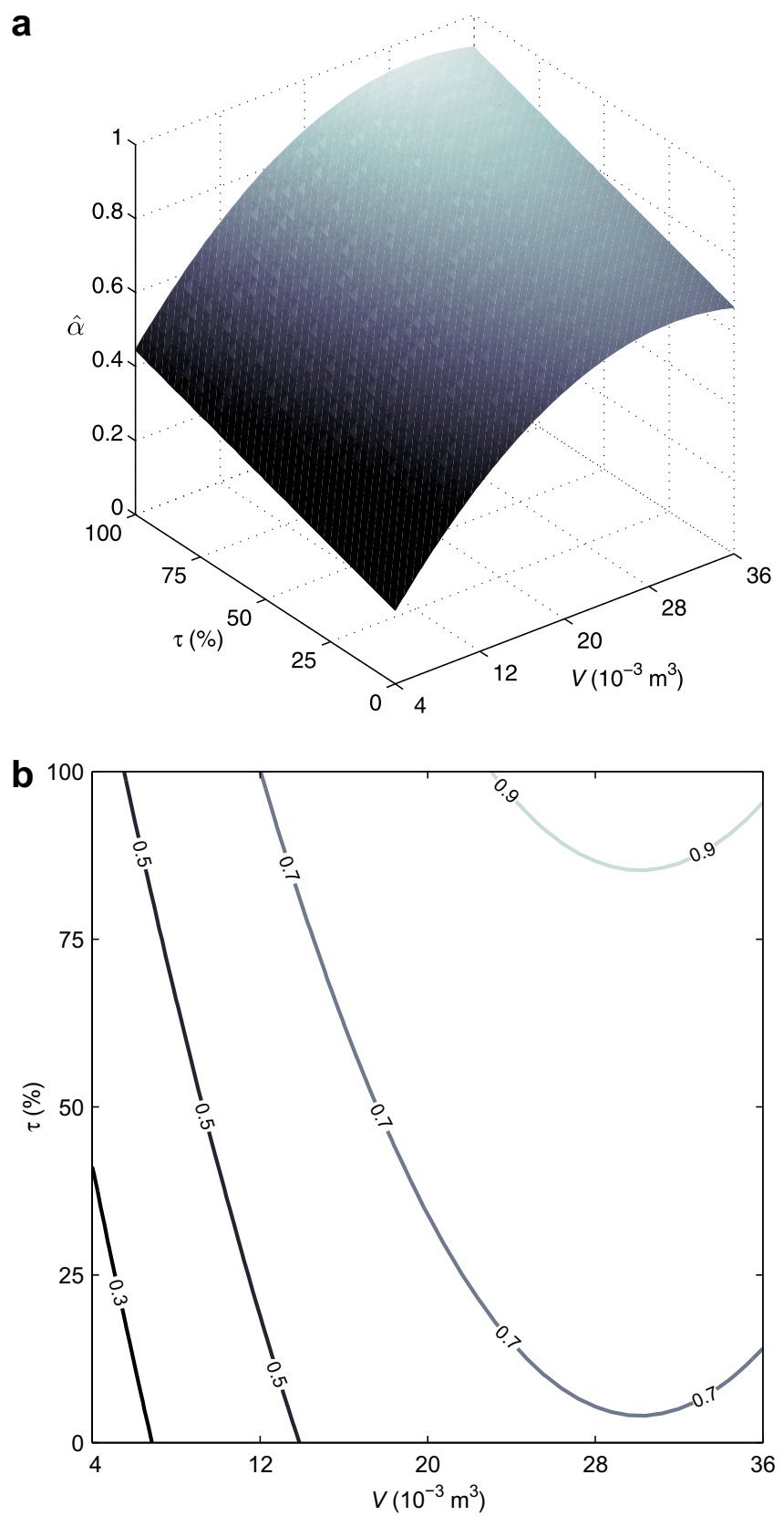

Fig. 16. Filling density vs. volume, $R=100 \Omega$ and $M=13 \times 10^{-3} \mathrm{~kg}$.

speaker is critical in order to design an effective sound absorber. Further development phases will now focus on the embodiment of an absorbing panel made of electroacoustic absorbers so as to obtain a large enough surface to significantly damp the first troublesome modes in the control room of the recording studio.

\section{Conclusion}

In this paper a method for optimizing acoustic performances of an electroacoustic absorber has been proposed. Assuming the change of a factor (or parameter) at a time often leads to select a wrong optimum, due to potential interactions between factors which are not taken into account, the response surface methodology has been applied to alleviate this situation. In this study four influential constitutive parameters have been selected to reflect some dissipative mechanisms of sound energy induced by an elec-

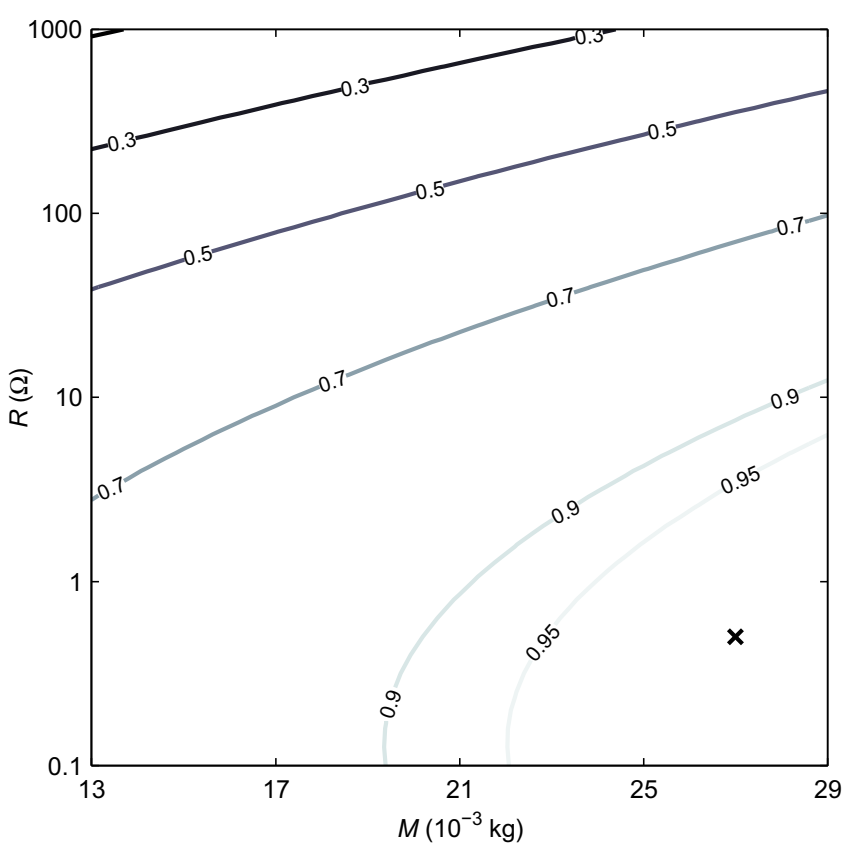

Fig. 17. Expected behavior for $V=10 \times 10^{-3} \mathrm{~m}^{3}$ and $\tau=0 \%$.

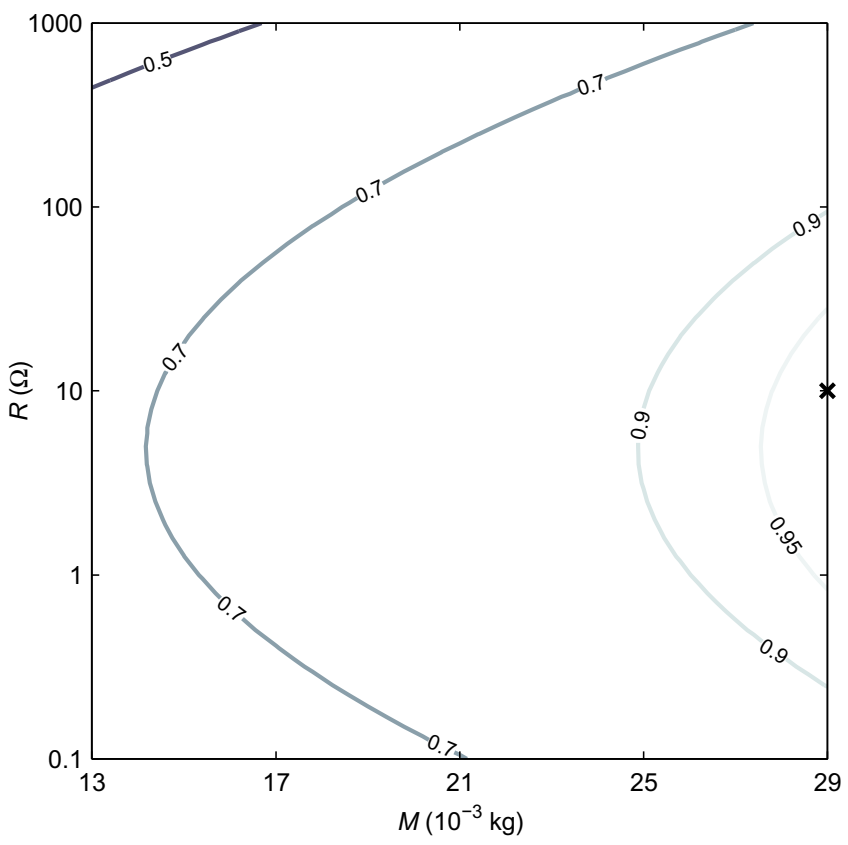

Fig. 18. Expected behavior for $V=10 \times 10^{-3} \mathrm{~m}^{3}$ and $\tau=80 \%$.

troacoustic absorber. Without much prohibitive computational efforts to process designed experiments, these methods based on approximation concepts show a real interest for optimizing a multivariate system such as an electroacoustic absorber, introducing statistics from upstream of the experimental process which increases the reliability of the results. From the series of tests carried out, several conclusions can be drawn. First, the information provided by RSM is helpful to give the direction of the design modifications and the fitted surfaces can be used to identify an appropriate direction of potential improvement for absorbing sound energy. Moreover the use of RSM can give a reliable insight into the estimation of the absorbtion coefficient anywhere within the space of the process. Results relate only to the $50 \mathrm{~Hz}$ one-third 


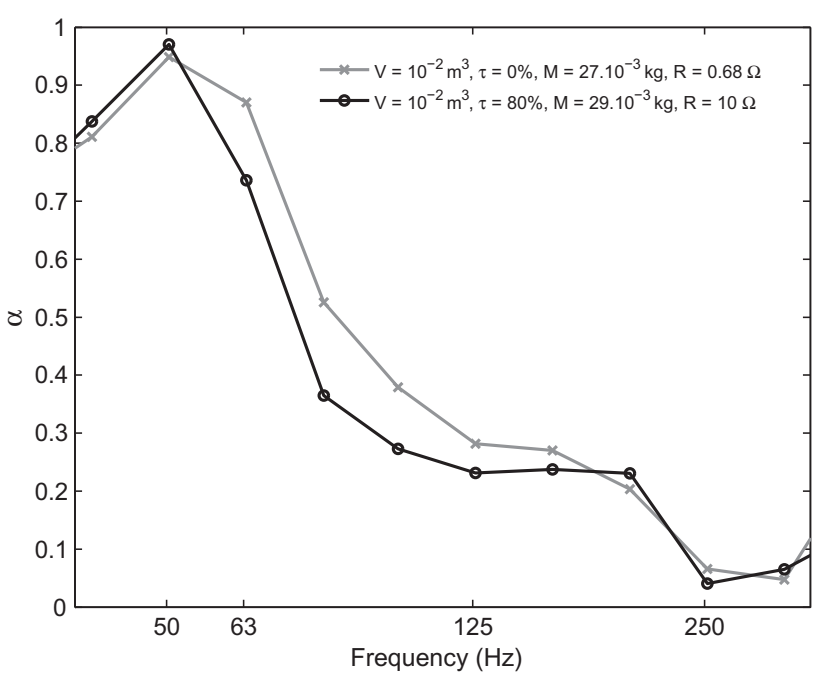

Fig. 19. Measured absorption coefficient after constrained optimization $V=$ $10 \times 10^{-3} \mathrm{~m}^{3}$.

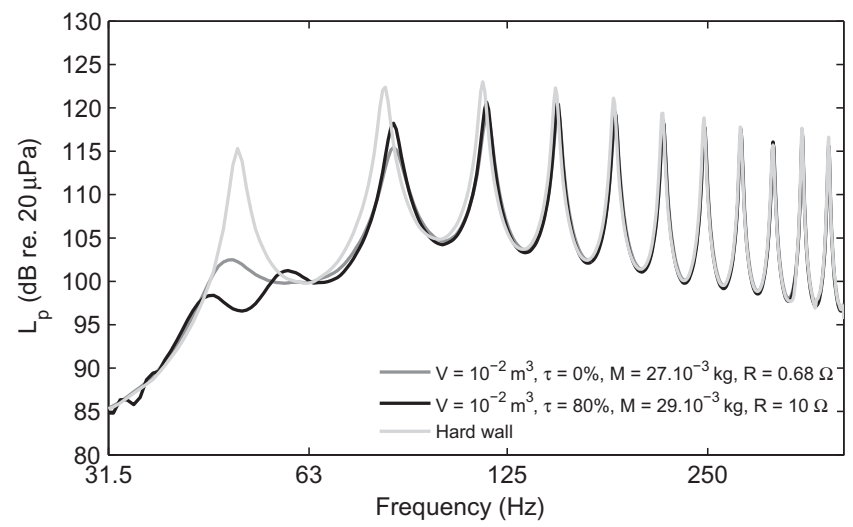

Fig. 20. Measured magnitude of the sound pressure in a duct.

octave band, but by using the same approach it is still possible to identify optimal operating conditions varying with frequency. Moreover, some unexpected effects can be easily highlighted. For instance, it has been shown that the optimal settings of the electroacoustic absorber drastically depends on the quantity of mineral fiber which fill the enclosure. With an empty enclosure the electrical load value needs to be lower than the d.c. resistance of the loudspeaker but nonzero, while conversely it needs to be upper in case of presence of mineral fiber inside the enclosure. Experiments performed after optimization on a closed acoustic duct demonstrate the effectiveness of electroacoustic absorber to attenuate the first resonant modes without affecting the response elsewhere. At last, it has been proven that, during the process of designing an electroacoustic absorber, the equivalent acoustic impedance of the backcavity should be taken into account in the models prior to compute the optimal electrical load to connect to the loudspeaker's electric terminals. This experimental technique could be especially useful for designing both electroacoustic transducer and its electrical and acoustical conditioning (enclosure, electric load, etc.) in a single process, without a priori selection of the loudspeaker. It can also alleviate the modeling of the numerous non-linear components of the transducer (suspension, driver) that could have potential prejudicial (or beneficial) effects on the performances on the electroacoustic absorber.

\section{Acknowledgments}

The authors wish to thank the Swiss National Science Foundation for supporting this work, under research Grant 200021116977.

\section{References}

[1] Lissek H. Les Absorbeurs Electroacoustiques: un Concept Unique pour les différentes Stratégies de Contrôle Actif d'Impédance Acoustique. In: Proceedings of the 10th French congress on acoustics; 2010.

[2] Fleming AJ, Niederberger D, Moheimani SOR, Morari M. Control of resonant acoustic sound fields by electrical shunting of a loudspeaker. IEEE Trans Contr Syst Technol 2007(4).

[3] Liu F, Horowitz S, Nishida T, Cattafesta L, Sheplak M. A multiple degree of freedom electromechanical Helmholtz resonator. J Acoust Soc Am, ASA 2007;122(1).

[4] Lissek H, Boulandet R, René P-J. Shunt loudspeakers for modal control in rooms. In: Proceedings of ICSV16; 2009.

[5] Yu G, Li D, Cheng L. Effect of internal resistance of a Helmholtz resonator on acoustic energy reduction in enclosures. J Acoust Soc Am, ASA 2008;124(6).

[6] Ruiz H, Cobo P, Siguero M. Optimization of multiple-layer microperforated panels by simulated annealing. In: Proceedings of inter-noise 2009, INCE; 2009.

[7] Box GEP, Wilson KB. On the experimental attainment of optimum conditions. J Roy Statis Soc Ser B (Methodologic) 1951;13(1).

[8] Hill WJ, Hunter WG. A review of response surface methodology: a literature survey. Technometrics 1966;8(4).

[9] Box GEP, Hunter WG, Hunter JS. Statistics for experimenters. New York: Wiley; 1978.

[10] Myers RH, Montgomery DC. Response surface methodology: process and product optimization using designed experiments. 2nd ed. Wiley-Interscience Publication; 2002.

[11] Liang X, Lin Z, Zhu P. Acoustic analysis of damping structure with response surface method. Appl Acoust 2007;68(9).

[12] Rossi M. Audio, Presses Polytechniques et Universitaires Romandes; 2007.

[13] ISO 10534-2: Acoustics - determination of sound absorption coefficient and impedance in impedance tubes - part 2: transfer-function method International Standard Organisation; 1998.

[14] Box GEP. Non-normality and tests on variances. Biometrika 1953;40(3/4).

[15] Snedecor GW, Cochran W. Statistical methods. IOWA State University Press; 1989.

[16] Archdeacon TJ. Correlation and regression analysis: an historian's guide. University of Wisconsin Press; 1994. 